\title{
Theory of Atomic-Scale Vibrational Mapping and Isotope Identification with Electron Beams
}

\author{
- SUPPLEMENTARY INFORMATION -
}

\author{
Andrea Konečná, ${ }^{1}$ Fadil Iyikanat, ${ }^{1}$ and F. Javier García de Abajo ${ }^{1,2, *}$ \\ ${ }^{1}$ ICFO-Institut de Ciencies Fotoniques, The Barcelona Institute of \\ Science and Technology, 08860 Castelldefels (Barcelona), Spain \\ ${ }^{2}$ ICREA-Institució Catalana de Recerca $i$ Estudis Avançats, \\ Passeig Lluís Companys 23, 08010 Barcelona, Spain
}

\section{SUPPLEMENTARY FIGURES}

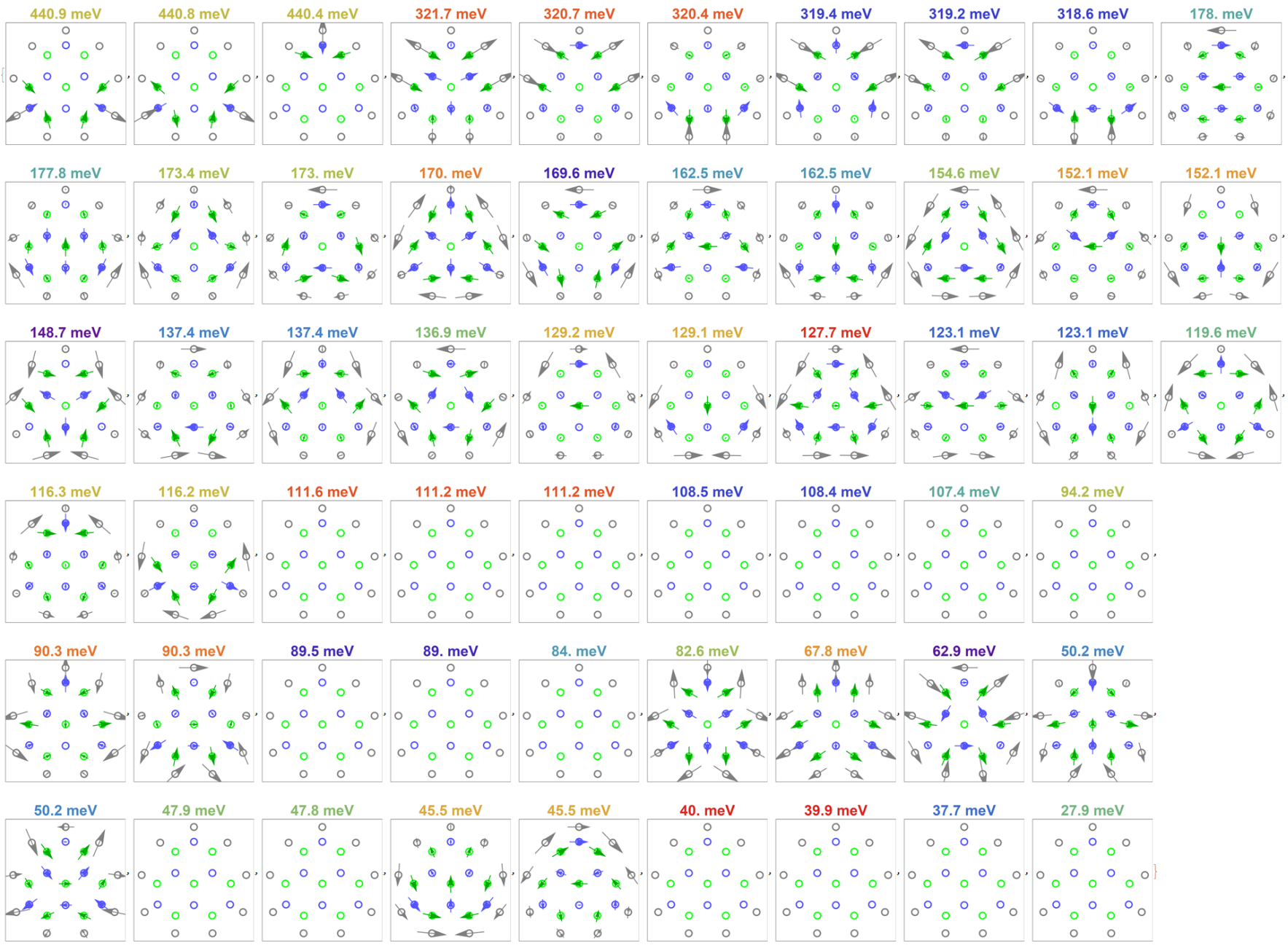

FIG. S1. Plots showing all of the eigenmodes and eigenvectors for the isotopically pure h-BN-like molecule in Figure 2a. Open circles denote the positions of B (green), $\mathrm{N}$ (blue), and $\mathrm{H}$ (gray) atoms, whereas the arrows show the magnitude and direction of the atomic displacement vectors projected on the plane of the atoms. Mode energies are indicated above the plots, with (quasi-)degenerate ones sharing the same label color. We have excluded the 9 lowest energy modes, 6 of which emerge from rigid translations or rotations of the molecule.

\footnotetext{
* javier.garciadeabajo@nanophotonics.es
} 


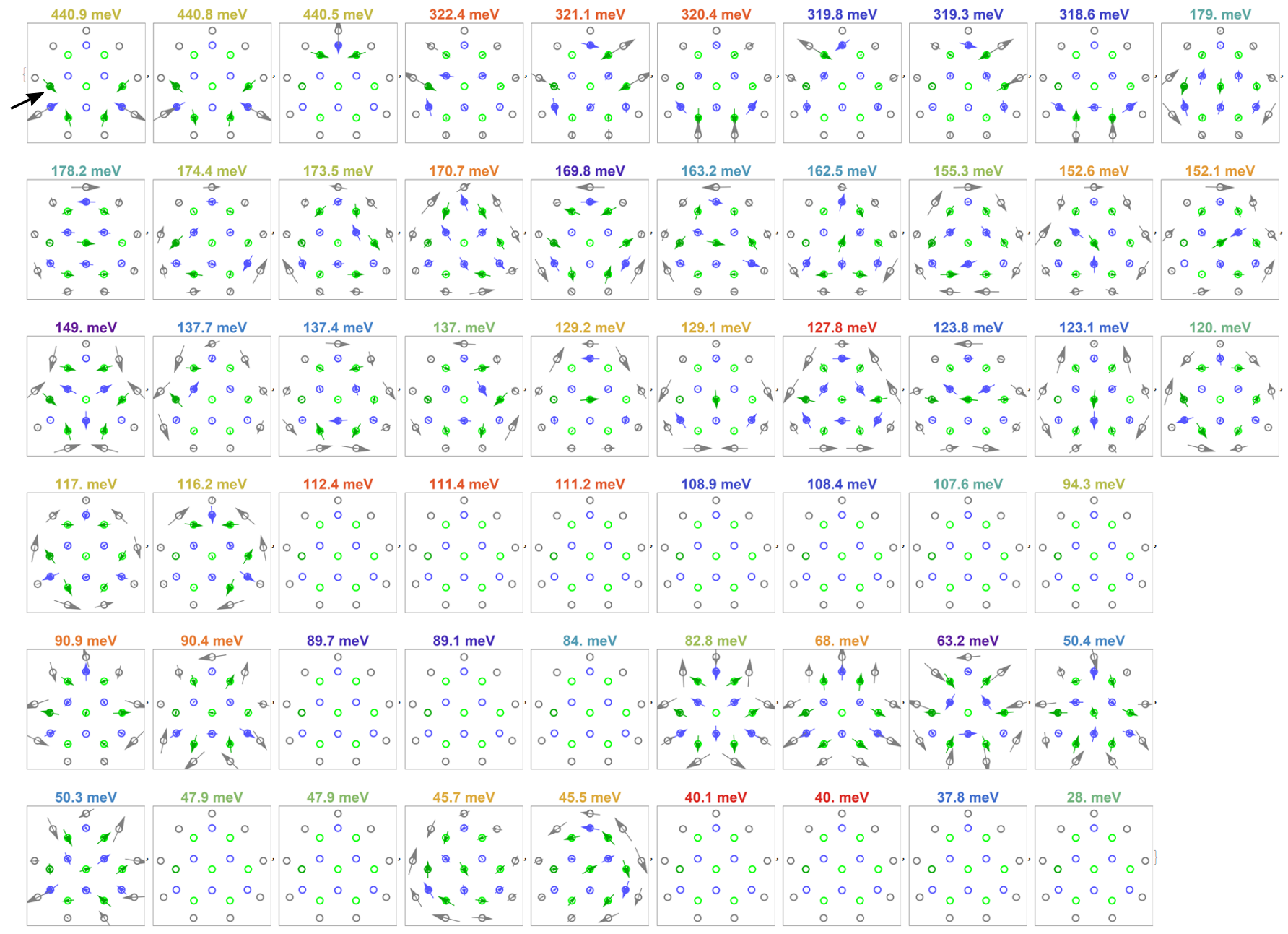

FIG. S2. Same as Fig. S1, but for the molecule containing a single B isotope impurity at the side of the molecule, represented by a dark green circle in each panel (see also black arrow in the upper-left panel). 


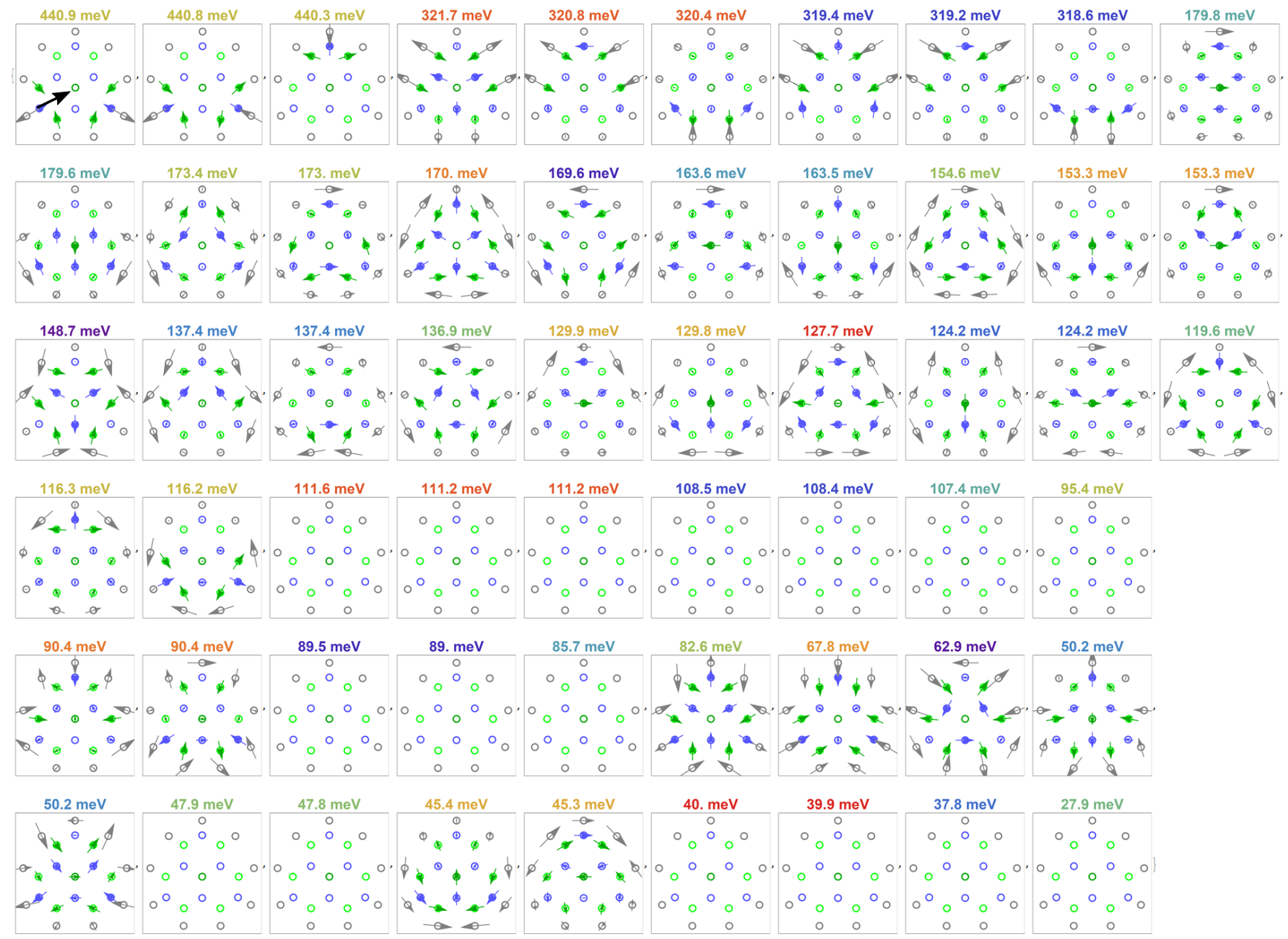

FIG. S3. Same as Fig. S1, but for the molecule containing a single B isotope impurity at its center, represented by a dark green circle in each panel (see also black arrow in the upper-left panel). 

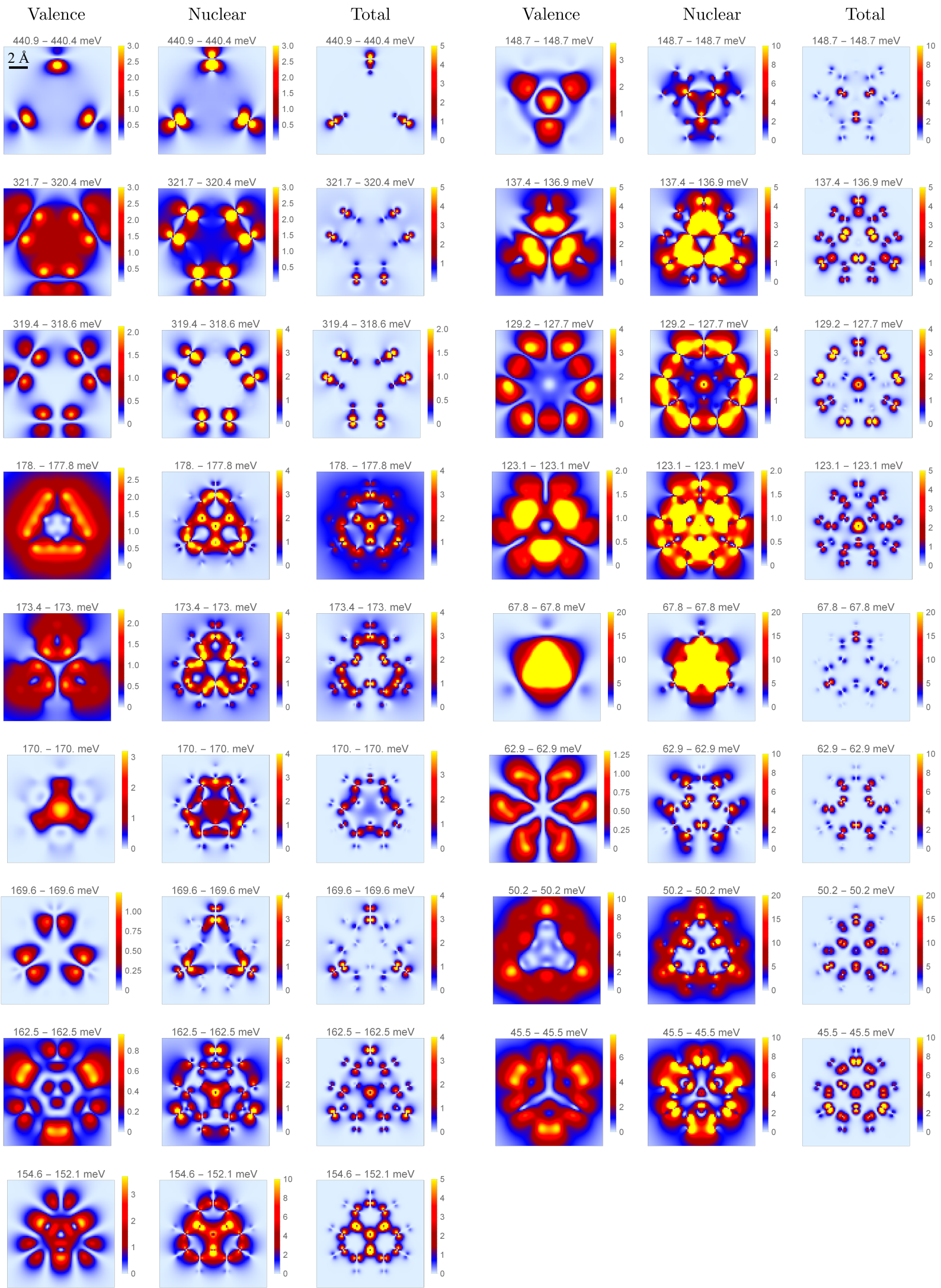

FIG. S4. Energy-filtered maps calculated as a function of the position of the focused electron beam by including different contributions to the polarization of the isotopically pure molecule associated with its vibrational modes (i.e., using the notation of the main paper, we consider partial contributions to $\vec{\rho}_{l}(\mathbf{r})=\vec{\rho}_{l}{ }^{\text {nucl }}(\mathbf{r})+\vec{\rho}_{l}{ }^{\text {val }}(\mathbf{r})$, where "nucl" refers to the sum of nuclear and core-electron charges, while "val" indicates the contribution of valence electrons). Different columns show calculations performed including valence-electron charges (valence), nuclear and core-electron charges (nuclear), and the sum of the two of them (total), as indicated by the upper labels. Each map is obtained by integrating the EELS probability over the indicated energy range. 

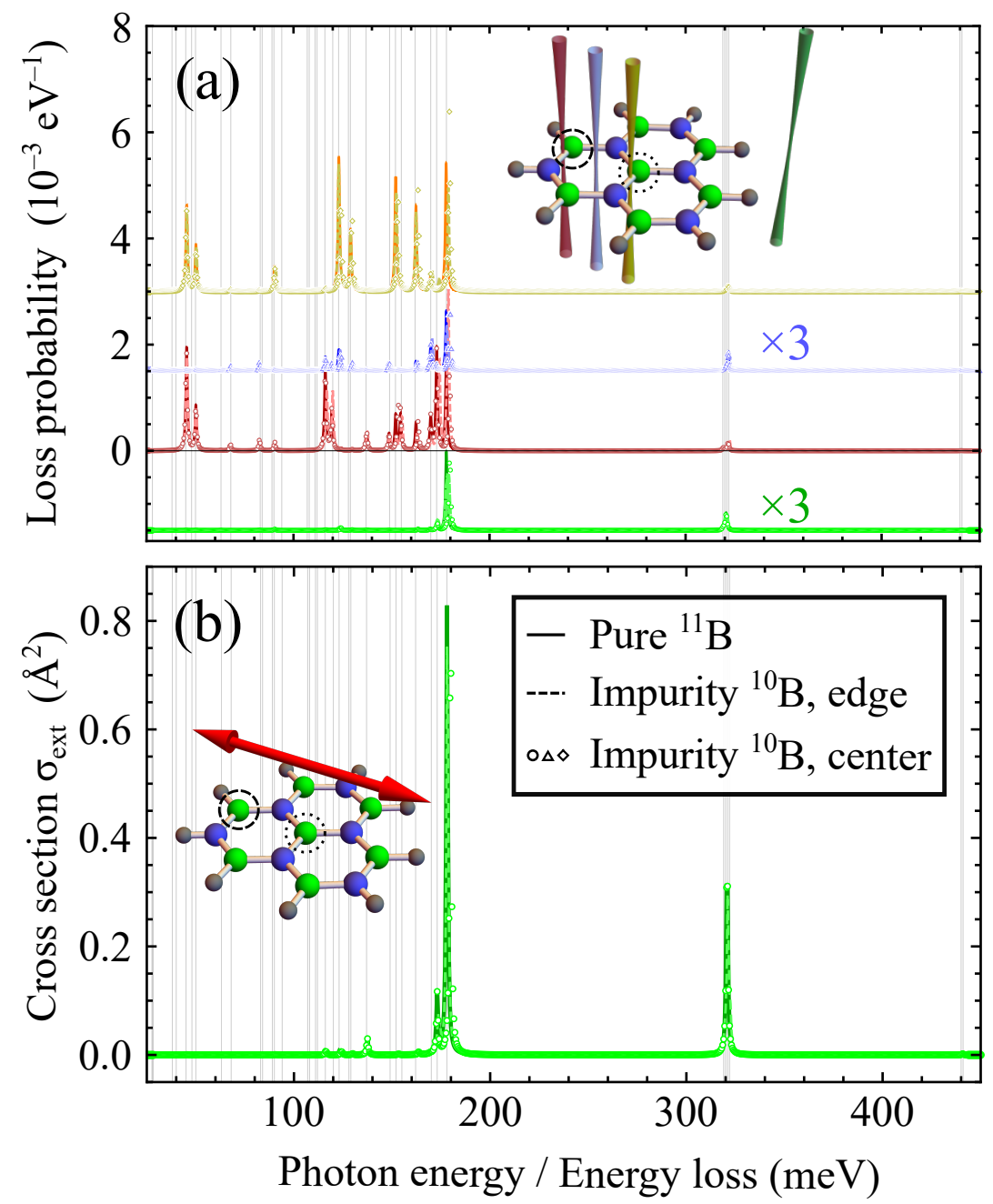

FIG. S5. Same as Figure 3a,c in the main text, but plotted over a wider energy range.

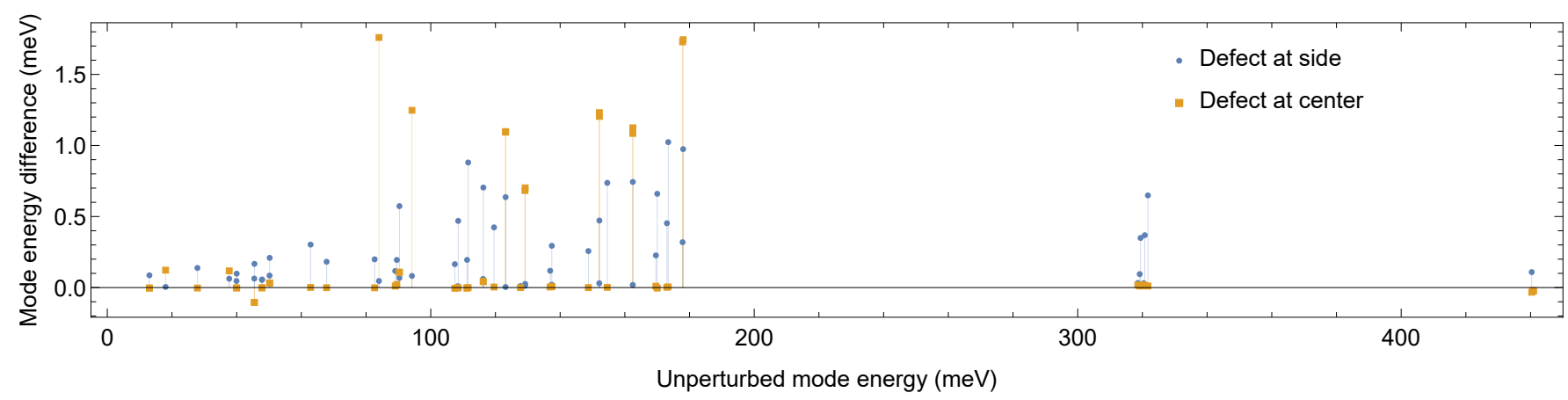

FIG. S6. Energy difference between the vibrational modes calculated for molecules with an isotope impurity (placed in either edge or center locations, see labels) relative to the isotopically pure molecule. The magnitude of the energy change can be linked to the amplitude of motion of the affected atom in the corresponding mode. For instance, breathing modes, in which the central atom does not move, are not affected by the presence of an isotope impurity at the center of the molecule. 
(a)

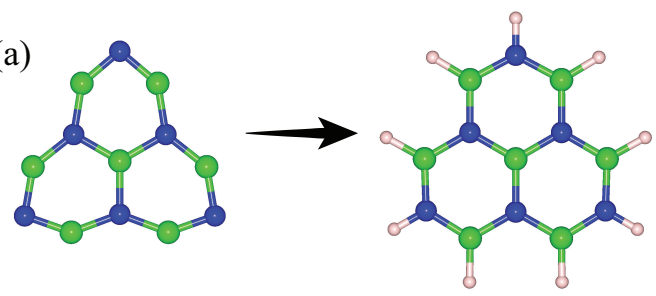

(b)

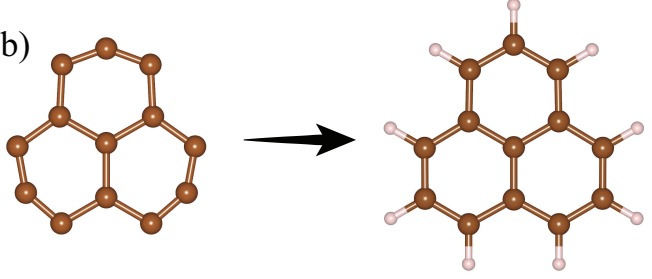

FIG. S7. Optimized atomic structure of (a) h-BN-like and (b) graphene molecules before (left) and after (right) passivation of the edges with additional hydrogen atoms. 